\title{
An Annotation System for 3D Fluid Flow Visualization
}

\author{
Maria M. Loughlin ${ }^{1}$ \\ Cambridge Research Lab \\ Digital Equipment Corporation
}

\author{
John F. Hughes ${ }^{2}$ \\ Department of Computer Science \\ Brown University
}

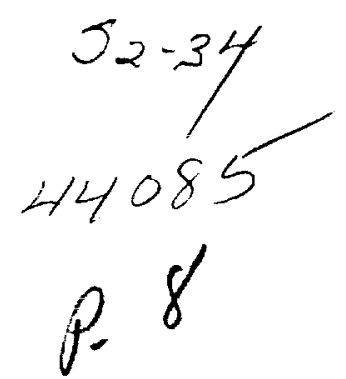

\begin{abstract}
Annotation is a key activity of data analysis. However, current systems for data analysis focus almost exclusively on visualization. We propose a system which integrates annotations into a visualization system. Annotations are embedded in 3D data space, using the Post-it ${ }^{3}$ metaphor. This embedding allows contextual-based information storage and retrieval, and facilitates information sharing in collaborative environments. We provide a traditional database filter and a Magic Lens ${ }^{4}$ filter to create specialized views of the data. The system has been customized for fluid flow applications, with features which allow users to store parameters of visualization tools and sketch 3D volumes.
\end{abstract}

\section{Introduction}

In a study to characterize the data analysis process, Springmeyer $e t$ al. [15] observed scientists analyzing different types of scientific data. The study found that recording results and histories of analysis sessions is a key activity of the data analysis process. In each session, the scientists recorded notes, and inspected previous notes. Two distinct types of annotating were observed:

- recording, or preserving contextual information throughout an investigation

and

- describing, or capturing conclusions of the analysis sessions.

Despite the importance of annotation, current systems for data analysis emphasize visualization, focusing on the

\footnotetext{
'One Kendall Square, Cambridge, MA 02139. email: loughlin@ crl.dec.com. phone: 617-621-6618

${ }^{2}$ Box 1910, Providence, RI 02912. email: jfh@cs.brown.edu. phone: 401-863-7638

${ }^{3}$ Post-it is a trademark of $3 \mathrm{M}$.

${ }^{4}$ Magic Lens is a trademark of Xerox Corporation.
}

generation of visual displays. Little or no annotation support is available: for example, Springmeyer $e t$ al. noted that the recording media used by scientists in their study included notebooks, scratch paper, and Post-it notes.

In this paper, we describe a system that supports annotation as an integrated part of a fluid flow visualization system. Unlike typical annotations on static 2D images, our system embeds annotations in 3D data space. This immersion makes it easy to associate user comments with the features they describe. To avoid clutter and data hiding, annotations are represented by graphical annotation markers that have associated information. Therefore graphical attributes of the markers, such as size and color, can be used to differentiate annotations with different functions, authors, creation dates, etc.

Annotations can easily be added, edited and deleted. Also, multiple sets of annotations can simultaneously be loaded into a visualization. This allows scientists, collaborating on a data set, to use annotations as a form of communication, as well as a history of data analysis sessions. Annotation markers also aid scientists in navigating through the data space by providing landmarks at interesting positions. Figure 1(a)-(c) shows the visualization environment, annotation markers, and the annotation content panel. Figure 1(d) shows a Magic Lens filter which hides the annotation markers and widget handles. The implementation has been applied to three-dimensional Computational Fluid Dynamics (CFD) applications. However, the techniques can be used in visualization systems of many disciplines. The design can also be extended to 3D stereo and virtual-reality environments.

The rest of this paper is organized in five sections. In section 2 we review previous approaches to annotation. Section 3 describes design guidelines for annotation systems. Section 4 details our implementation of annotation within a 3D modeling and animation system. In the last two sections, we discuss possible future work and present our conclusions.

\section{Background}

Scientific visualization systems provide little, if any, support for annotation. For example, Application Visualization Sys- 


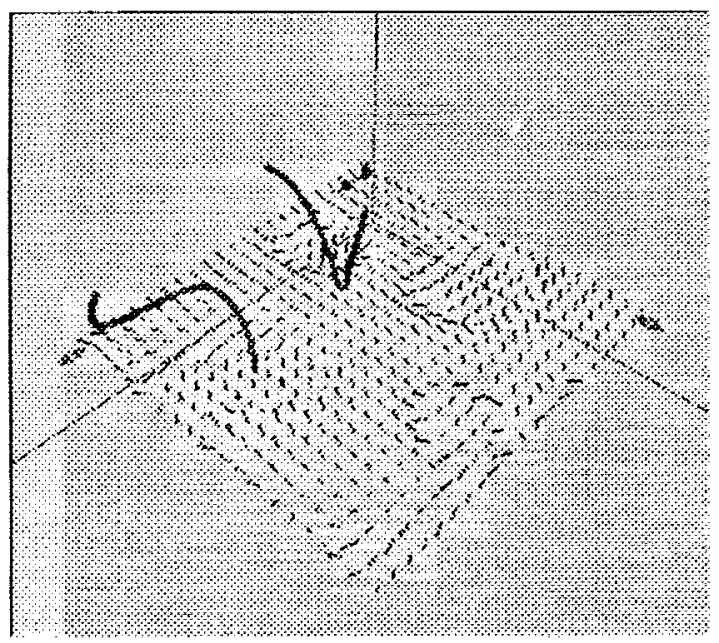

(a)

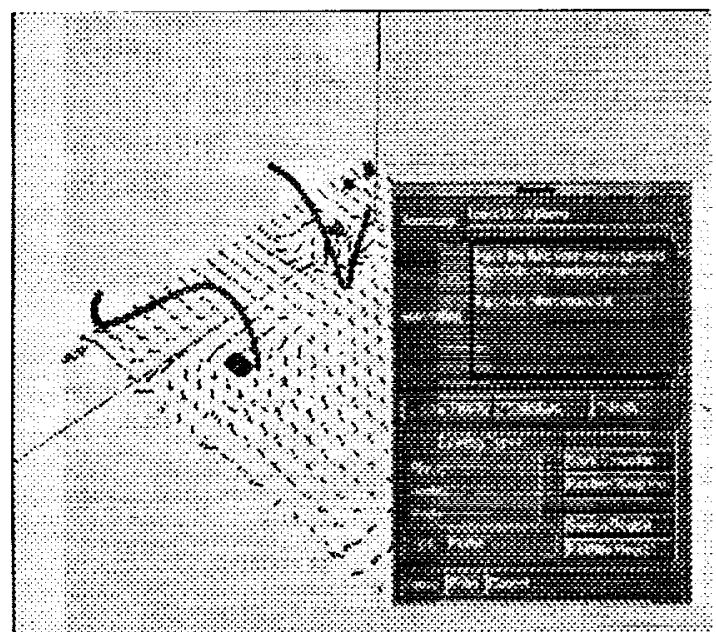

(c)

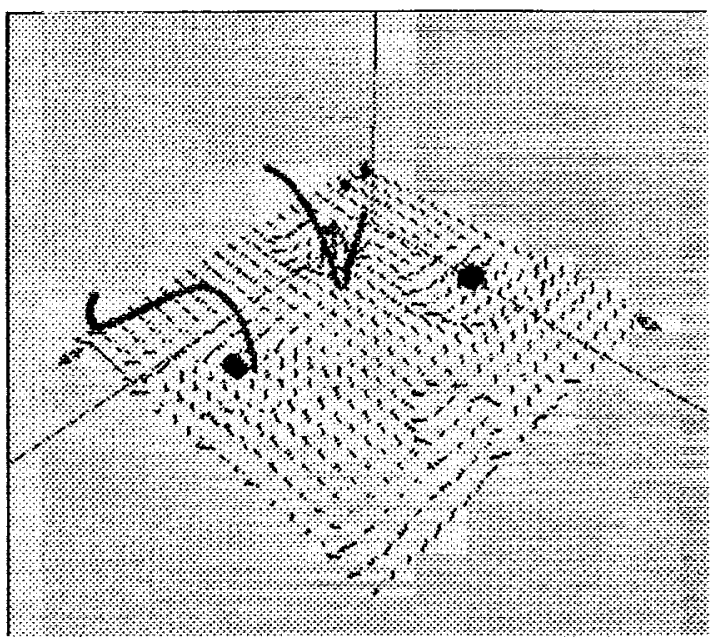

(b)

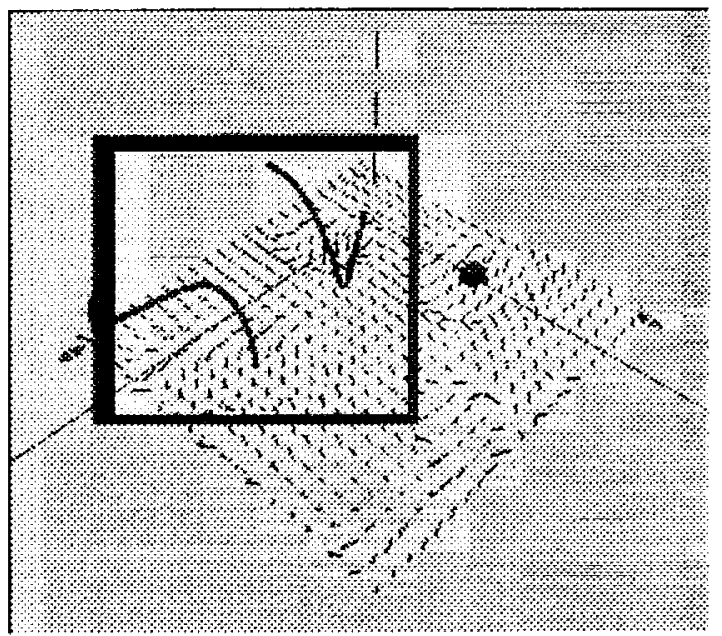

(d)

Figure 1: The visualization and annotation system

(a) hedgehog and streamlines showing 3D fluid flow, (b) annotation markers (small geometric objects) placed at points of high velocity, (c) annotation content panel, (d) Magic Lens filter hiding annotation markers and widget handles. 
tem (AVS) [17] and Flow Analysis Software Toolkit (FAST) [1], two software environments for visualizing scientific data, facilitate attachment of labels to static $2 \mathrm{D}$ images. These systems also allow a user to record a sequence of interactions with the visualization. This support is useful for generating presentations from the data, but does not facilitate the recording and describing operations observed by Springmeyer $e t$ al.

Outside the scientific visualization domain, annotations of various sorts have been integrated in different applications.

MacDraw, a 2D paint program, introduced a notes feature, which allows static 2D annotations using the Post-it metaphor. Media View [12] [13], a multi-media publication system, extends the conventional paradigm of a document and allows annotations in all media components including text, line art, images, sound, video sequences, and computer animations. The format of annotations has been expanded, but their use is still limited to presentation of information in a static environment.

Document annotation is used as a means of communication in the Wang Laboratories multi-media communication system, Freestyle [8]. Freestyle's multi-media messages are based on images, including screen snapshots and hand-drawn sketches. Furthermore, this system allows synchronization of input modalities, such that messages can contain information about the process by which they were created. Freestyle advances the concept of annotations as communicators, but does not address the issues of clutter and management of annotations in the environment.

Verlinden $e t$ al. [18] developed an annotation system to explore communication in Virtual Reality (VR) environments. In general, annotation in immersive VR systems is restricted, as the user must interrupt the session to interact with objects in the real world, such as notebooks and computer monitors. Verlinden's system overcomes this problem by embedding verbal annotations in the VR space. The annotations are represented as visual 3D markers. When the user activates a marker, the verbal message stored with that marker is played. This system is unique in that it embeds annotations in 3D scenes, but it is limited to verbal annotations and provides no support for annotation filtering. It also limits annotations to a fixed position in a time-based environment.

\section{Design Issues}

We have extracted, both from the Springmeyer et al. study and from our own experience with scientific visualization, a set of three design guidelines that seem appropriate for an annotation system. These guidelines, discussed below, formed the basis for the design of our system.

Guideline 1: To support ongoing recording of contextual information, an annotation system must be an integral part of a visualization system. Effective placement and storage of annotations are required.

Traditionally, annotations to scientific visualizations are recorded on paper or in electronic files, and both the dataset and the files are labeled to mark their association. This separation of data and annotations means that some effort is required to find the data features described by annotations. The 3D data space of many scientific applications provides the context in which annotations should be placed. Recording annotations in this space capitalizes on human's spatial senses by facilitating the retrieval of information based on its spatial location in the visualization.

However insertion of annotations in the data space creates an immediate conflict between the annotation and visualization functions: both compete for screen territory. We do not wish to impose any restrictions on the amount of information that can be recorded. At the same time, since information is contained in the data itself, we do not wish data to be obscured by annotations. Our approach is to decompose an annotation into:

- an annotation marker or small geometric object that identifies the position of the annotation in the data space and

- an annotation content in which a user stores information.

The geometry and graphical attributes of markers are chosen so that they are easily distinguished from existing visualization tools. By clicking on a marker, a user can expand the associated annotation to read or edit its content. Separation of the annotation's content from the annotation marker in this way allows direct insertion of arbitrarily large annotations.

Guideline 2: Annotations must be powerful enough to capture information considered important by the user.

There are different types of information. Tanimoto [16] distinguishes between data (raw figures and measurements), information (refined data which may answer the users' questions) and knowledge (information in context). Bertin [3] classifies the levels of information in a similar way. He considers information as a relationship which can exist between elements, subsets or sets. The broader the relationship, the higher the level of information. We assume that an annotation system should be able to store information at each of these levels - scientists peed to record both the data values at probe points in the data set, and a higher-level analysis of these figures.

Although some data, such as date of creation and author, are likely to be relevant to all applications, it is possible that knowledge can be captured only when an annotation system is customized for a specific application. The customization would ensure that annotations can represent information relevant in the context of the application. For example, if the data of a particular application is time-varying, the annotation system should provide time-varying annotations that can track the features being described.

In our annotation system, we provide support for different types of information in two ways. First, within each 
annotation, scientists can record both numerical and textual details, and high-level information specific to fluid flow. This is discussed in section 4.4. Second, the system supports hierarchically-organized annotations. The hierarchical structure allows scientists to record facts in separate annotations, and group related annotations in sets that describe broader observations.

It is also important to consider the modalities that are available for capturing information in an annotation system. Two dimensional text, graphics and images are the standard annotation modalities; aural annotation is also a candidate. Chalfonte, in an experiment on the use of annotation for collaborative document authoring, found aural annotations a richer and more effective medium for high-level communication [5]. Freestyle shows that coordinating hand/cursor movements with textual and aural annotations is also effective.

In our current implementation, we use 2D text and 3D volumes to store information. In the future, we would like to use different interaction techniques for information capture.

Guideline 3: We need to consider the established rules of user interface (UT) design, because the UI of an annotation system will play a key role in determining its acceptance (or lack thereof) by scientists.

We considered many UI rules [7] and designed our annotation system accordingly. One rule states that a UI should allow users to work with minimal conscious attention to its tools. We achieve this goal by using a direct manipulation interface, that is, an interface in which the objects that can be manipulated are represented physically. For example, the volume of data affected by the Magic Lens filter can be controlled directly by moving and resizing the physical representation of the lens. Another design rule states that an interface should provide feedback, e.g., on the current settings of domain variables. In our system, annotation markers give visual feedback on the location of annotations and marker geometry gives feedback on annotation content.

Because the geometric data space of fluid flow applications has three dimensions, we considered design issues specific to 3D graphical user interfaces [6]. One issue is the complexity introduced by 3D viewing projections, visibility determination, etc. A second issue is that the degrees of freedom in the 3D world are not easily specified with common hardware input devices. A third issue is that a $3 D$ interface can easily obscure itself. We use guidelines outlined by Snibbe et al. [14] to deal with these problems. For example, we provide shadows, constrained to move in a plane, to simplify positioning of annotation markers (see section 4.3.2). We provide feedback on the orientation of the data by optionally drawing the principal axes and planes. We also ensure that annotations do not obscure data, by making it easy for a user to change the viewpoint and resize or hide annotation markers.

\section{Implementation}

This section describes the annotation system we have implemented. We begin by setting a context for the system with a description of fluid flow visualization and the software development environment. Then we discuss the main components of the annotation system: the annotation markers, support for information capture, and interaction techniques.

\subsection{Fluid Flow Visualizations}

Computational fluid dynamics (CFD) uses high speed computers to simulate the characteristics of flow physics. Computed flow data is typically stored as a 3D grid of vector and scalar values (e.g., velocity, temperature, and vorticity values), which are static in a steady flow, and change over time in an unsteady flow. CFD visualization tools allow a scientist to examine the characteristics of the data with 3D computer displays.

Interaction with the visual representation is essential in the exploration and analysis of the data, and has three goals: feature identification, scanning, and probing [9]. Feature identification techniques help find flow features over the entire domain, and give the scientist a feel for the position of interesting parts of the flow volume. An example of this type of technique is a vector hedgehog, a three-dimensional array of velocity vectors. Scanning techniques are used to interactively search the domain, by varying one or more parameters, through space or through scalar and vector field values. Scanning techniques include cutting planes (planar surfaces which slice the 3D grid and show scalar field value at each grid point of the plane) and iso-surfaces (three-dimensional surfaces of a constant scalar value). Probing techniques are localized visualization tools, typically used to gather quantitative information in the final step of investigating a flow feature. Examples of probing tools include streamlines and particle paths, which show the path in which a particle would flow if positioned in a steady or unsteady fluid flow.

The Computer Graphics Group at Brown University has developed a flow visualization system, to study new modes of interaction with flow tools. The annotation system was developed as part of this flow visualization system. This provided a test-bed for techniques to integrate visualization and annotation functionality .

\subsection{The Development Environment}

The annotation system was developed using FLESH, an object oriented animation and modeling scripting language [11], and $\mathrm{C}++$. In the FLESH language, scenes are described as collections of objects. The FLESH objects defined for the annotation system include geometric objects such as annotation markers, 3D volumes and lenses, and non-geometric objects, such as holders for collections of annotations and an annotation filter. Some of these FLESH classes have corresponding $\mathrm{C}++$ classes, in which data is stored and compute-intensive operations performed. This allows us to benefit from the 
power of an interpreted interactive prototyping modeling system and the efficiency of a compiled language.

\subsection{Annotation Markers}

Annotations are represented in the 3D data space by small geometric markers. Each marker has an associated content which the user can edit at any time.

\subsubsection{Marker Geometry and Graphical Attributes}

The geometry of a marker gives visual feedback on the content of the annotation. In the fluid flow visualizations system, the user can define annotation keywords (e.g., plume, vortex), and select a geometry to associate with each keyword. Then, when the user assigns a keyword to an annotation in the system, the annotation's marker takes the associated shape. It is likely that other mappings between graphical attributes of markers and annotation content would also be useful. For example, the color saturation of a marker could depend on the age or priority of the annotation.

The graphical attributes of annotations are also usercustomizable. The size and color of all markers in one level of hierarchy can be changed. We predict that this feature would be useful if many scientists work collaboratively on a data set, and each scientist defines a unique color and size for her markers.

\subsubsection{Marker Behavior}

Since the function of a marker is simply to identify points of interest in the visualization, its behavior is quite simple. A marker is created when the user presses the annotation push-button. It appears at the point on which the user is focussed, making it easy for the user to position it near the feature of interest.

The scientist can translate and rotate markers with simple mouse movements. He can also project interactive shadows of the marker on the planes defined by the principal axes [10]. Each shadow is constrained to move in the plane in which it lies. If a user moves a shadow, the marker moves in a parallel plane. This constrained translation helps in precisely positioning a marker.

Markers can be highlighted in response to a filter request. In the current system, the color of a marker changes to a bright yellow when highlighted. This simple approach seems adequate. However, the user may change this highlight behavior, by, for example, having highlighted markers flash between alternating colors.

Since the features of unsteady fluid flows change over time, a user would like the annotation describing a particular feature to follow the feature's movement in the visualization. The current annotation system provides partial support for this by allowing the user to specify the position of an annotation at any number of points in time. The annotation markers then linearly interpolate between the specified positions in time.

\subsection{Knowledge Stored}

Our annotations can store generic information, as well as information specific to fluid flow applications. The generic information includes keyword, textual summary and description, author, and date. Some of this information (author and date) are captured implicitly when the annotation is created. The rest must be explicitly added after the scientist has opened the annotation by clicking on it. This data entry is performed via a 2D Motif panel of buttons and text widgets. We consulted with fluid flow experts to understand how the information content of annotations could be customized for fluid flow applications.

\subsubsection{Parameters of Visualization Tools}

One of the key additions to the annotation system suggested by the fluid flow experts results from the interactive nature of fluid flow analysis. As described earlier, a scientist must insert flow visualization tools (such as streamlines and iso-surfaces) in the data space to see the underlying data. Much time is spent determining which tools most effectively highlight a feature, and positioning and orienting both the tools and viewpoint to best show off the feature being described. Springmeyer $e t$ al. observed this activity of the data analysis process, and described it as orientating the data, or altering a representation to gain perspective.

To support this activity, our concept of an annotation was expanded to include parameters of flow visualization tools. When a user wishes to store the parameters of a set of tools, he or she presses a button to indicate that a set of tools is being saved, and then clicks on the tools of interest. The timevarying location, orientation, size, and other parameters of the tools are saved with the annotation. This can be repeated any number of times for different groupings of tools with different parameters. When an annotation is restored, the user is presented with a list of all saved sets of tools, and can recover each set of tools to see how they illustrate the annotated feature.

\subsubsection{D Volume Descriptions}

It also became obvious that annotation markers, which are appropriate for locating point features in a visualization, are not sufficient for CFD applications. Fluid flows contain volume features, such as vortices (masses of flow with a whirling or circular motion) and plumes (mobile columns of flow). Users may want to associate an annotation with a region of the data space, rather than a single point in the space. We therefore need a way to sketch a volume in the data space. The volume-sketching method must be intuitive, so that flow scientists (who may not be interested in becoming artistic volume sculptors!), can easily describe the volume. Also, the resolution of the volumes sketched need only be as precise as the grid on which the flow field is defined.

We provide a simple method to sketch volumes. The user positions "pegs" that define the extreme vertices of the volume to be drawn. The pegs are created and moved within 


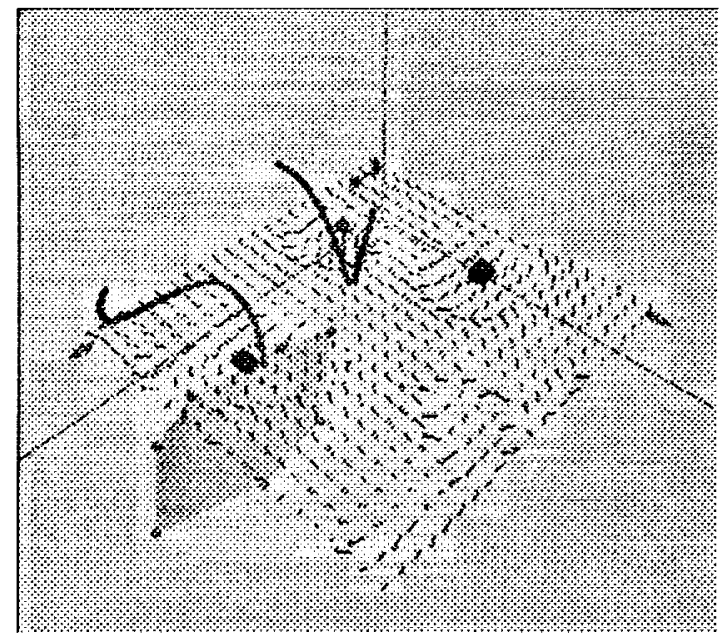

Figure 2: A volume defined as the convex hull of a set of pegs.

the visualization in a way similar to the creation and translation of annotation markers. When the user is done positioning pegs, the system computes their convex hull using the quickhull algorithm [2]. The boundary of the volume, defined by the convex hull, is rendered in either wireframe or transparent mode. Vertices can be added, deleted and moved, and the volume redrawn, until the volume is accurate. Figure 2 shows a volume which has been defined in this way.

This implementation provides a simple means to draw volumes. However, since it uses the convex hull of the pegs, certain shapes, such as a 3D " $\mathrm{L}$ " shape, cannot be sketched.

\subsection{Retrieving the Annotations}

Effective information retrieval and communication requires that a user can easily identify annotations relating to a specific topic, by a specific author, etc. The annotation system facilitates such data filtering in two ways.

First, a traditional database filter is provided. The user can specify data selection criteria (such as the annotation creation date, author, or keyword), via a 2D Motif panel. The markers of annotations that satisfy the search criteria are highlighted.

A second filter uses the Magic Lens metaphor introduced by Bier $e t$ al. [4]. A Magic Lens filter is a rectangular frame, placed in front of the visualization, that appears as if it moves on a transparent sheet of glass between the display and the cursor. The lens performs some function (which may use information from application-specific data-structures) on the application objects behind it.

Four functions are defined for the lens in the annotation system. The first sets the color of all objects, except annotation markers, to gray. This helps users find markers in a cluttered scene. The second lens function displays only the annotations that satisfy the criteria specified in the Motif database filter. The third lens function hides all annotation markers behind the lens. Finally, the default function hides all annotation markers and all interaction handles on the visualization tools behind the lens. Many other interesting lens . functions could be defined. One such function could remove all fluid flow tools except those in the user sketched volume behind the lens.

We believe that the magic lens filter alleviates the problem of visualization and annotation functions sharing the same screen space. Using the lens, a scientist can tightly integrate the two functions when appropriate. When she wishes to focus exclusively on either visualization or annotation, the clutter introduced by the other component can be hidden.

\section{Future Work}

The work described in this paper could be expanded in a number of ways, in both the fluid flow application and in new environments.

There are a number of opportunities for the fluid flow application. The facility for recording parameters of visualization tools could be extended to record view parameters. Then, flow tools could automatically be viewed from the same viewpoint and with the same magnification as when their parameters were saved. Annotations could also become more active in the data investigation process. For example, annotation markers could be used as seed points for automatic flow feature-characterization code. The output of the feature-characterization code (i.e., specifications of the feature found) could then be added to the annotation content. Feature-characterization code could also be used to improve support for time-varying annotations. If the location of an annotation marker were constrained to the feature's position (as found by feature-characterization code), the marker would follow the movement of the feature over time.

We would also like to implement annotations in other applications and environments. For example, virtual reality environments pose many new research problems. User studies would have to be performed to determine which annotation modalities would be appropriate in this space. If textual annotations were appropriate, we would have to determine where to place the text: floating in space near the marker, or on 2D panels which exist in the virtual space, or perhaps in some other place. New interaction mechanisms for annotation markers and filters should also be developed.

Finally, we would like to expand the scope of annotations. Springmeyer $e t$ al. noted that scientists tend to record their interactions with visualization systems. Perhaps the annotation system could help in recording and examining these edit trails. Also, scientists spend time comparing different data sets. The current annotation system could be redesigned to fit in the context of more than one data set.

We hope that further experience with the current system and its extension to other applications and environments will allow us to evaluate our design guidelines, and develop principles for customization of a general purpose annotation system. 


\section{Conclusion}

The importance of annotation in data analysis and the lack of annotation support in data analysis tools led us to develop a system that integrates annotation and visualization. In our system, annotations are embedded in the 3D space of CFD data. Co-location of annotations and data allows users to navigate through the information by spatial association. Each annotation is composed of a small geometric marker and a content that can include textual, graphical and other domainspecific information. This allows unobtrusive annotations with an unlimited amount of information. Filters are provided to help sort annotations and create customized views of the information.

Initial feedback from scientists leads us to believe that the close integration of annotation and visualization facilitates the ongoing recording activity observed by Springmeyer $e t$ al. At the same time, the ability to group annotations in disjoint sets and filter annotations supports the organization of analysis conclusions, i.e., the describing activity. Furthermore, annotations can be used as a means of communication between collaborating scientists, and as a way to present information in an educational tool.

\section{Acknowledgments}

The authors of this paper would like to thank the members of the Graphics Group at Brown for their helpful comments and support. Thanks also to the members of the Visualization group at Digital Equipment Corporation's Cambridge Research Lab for their review of this paper. The paper is based on the Master's thesis of the first author, whose attendance at Brown University was made possible by Digital Equipment Corporation's Graduate Engineering Education Program. This work was supported in part by grants from Digital Equipment Corporation, NSF, DARPA, IBM, NCR, Sun Microsystems, and HP.

\section{References}

[1] Bancroft, Gordon V., Merritt, Fergus J., Plessel, Todd C., Kelaita, Paul G., McCabe, R. Kevin, and Globus, Al. FAST : A Multi-Processed Environment for Visualization of Computational Fluid Dynamics. Proceedings of the First IEEE Conference on Visualization, pages 14-27, 1990.

[2] Barber, C. Bradford, Dobkin, David P., and Huhdanpaa, Hannu. The Quickhull Algorithm for Convex Hull. Technical Report GCG53, Geometry Center, U. Minnesota, July 1993.

[3] Bertin, Jacques. Graphics and Graphic Information Processing. Walter de Gruyter and Co., 1981.

[4] Bier, Eric A., Stone, Maureen C., Pier, Ken, Buxton, William, and DeRose, Tony D. Toolglass and Magic Lenses: The See-Through Interface. Proceedings of SIGGRAPH '93, pages 73-80, 1993.
[5] Chalfonte, Barbara L., Fish, Robert S., and Kraut, Robert E. Expressive Richness: A Comparison of Speech and Text as Media for Revision. Proceedings of the ACM Computer Human Interaction Conference, pages 21-26, 1991.

[6] Conner, D. Brookshire, Snibbe, Scott S., Herndon, Kenneth P., Robbins, Daniel C., Zeleznik, Robert C., and van Dam, Andries. Three-Dimensional Widgets. Proceedings of the Symposium on Interactive 3D Graphics, pages 183-188, 1992.

[7] Foley, James, van Dam, Andries, Feiner, Steven, and Hughes, John. Computer Graphics Principles and Practice. Addison W esley, 2nd edition, 1992.

[8] Francik, Ellen, Rudman, Susan E., Cooper, Donna, and Levine, Stephen. Putting Innovation to Work: Adoption Strategies for Multimedia Communication Systems. Communications of the ACM, 34(12):53-63, Dec. 1991.

[9] Haimes, Robert and Darmofal, Dave. Visualization in Computational Fluid Dynamics: A Case Study. Proceedings of the Second IEEE Conference on Visualization, pages 392-397, 1991.

[10] Herndon, Kenneth P. Interactive Shadows. UIST Proceedings, pages 1-6, November 1992.

[11] Meyer, Tom and Huang, Nate. Programming in FLESH. Technical report, Department of Computer Science, Brown University, 1993.

[12] Phillips, Richard L. An Interpersonal Multimedia Visualization System. IEEE Computer Graphics and Applications, pages 20-27, May 1991.

[13] Phillips, Richard L. MediaView, A General Multimedia Digital Publication System. Communications of the ACM, 34(7):74-83, July 1991.

[14] Snibbe, Scott S., Herndon, Kenneth P., Robbins, Daniel C., Conner, D. Brookshire, and van Dam, Andries. Using Deformations to Explore 3D Widget Design. Proceedings of SIGGRAPH '92, pages 351-352, 1992.

[15] Springmeyer, Rebecca R., Blattner, Meera. M., and Max, Nelson. L. A Characterization of the Scientific Data Analysis Process. Proceedings of the Second IEEE Conference on V isualization, pages 351-352, 1992.

[16] Tanimoto, Steven L. The Elements of Artificial Intelligence. Computer Science Press, 1990.

[17] Upson, C. and et al. The Application Visualization System: A Computational Environment for Scientific Visualization. IEEE Computer Graphics and Applications, 9(4):60-69, July 1989. 
[18] Verlinden, Jouke C., Bolter, Jay David, and van der Mast, Charles. Voice Annotation: Adding Verbal Information to Virtual Environments. Proceedings of the European Simulation Symposium, pages 60-69, 1993. 